Notfall Rettungsmed 2020 $23: 578-586$ https://doi.org/10.1007/s10049-020-00759-8 Online publiziert: 15 . Juli 2020

(c) Springer Medizin Verlag GmbH, ein Teil von Springer Nature 2020, korrigierte Publikation 2020

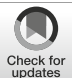

Michael Dommasch ${ }^{1}$ Friedemann Gebhardt ${ }^{2} \cdot$ Ulrike Protzer $^{3} \cdot$ Angelika Werner $^{4}$. Eva Schuster ${ }^{5}$. Christoph Brakemeier ${ }^{6} \cdot$ Julia Mayer $^{6} \cdot$ Susanne Feihl ${ }^{7}$. Christiane Querbach ${ }^{8} \cdot$ Rickmer Braren $^{9} \cdot$ Matthias Treiber $^{10} \cdot$ Fabian Geisler $^{10}$. Christoph D. Spinner"

'Fakultät für Medizin, Zentrale Interdisziplinäre Notaufnahme, Klinikum rechts der Isar, Technische Universität München, München, Deutschland; ${ }^{2}$ Fakultät für Medizin, Krankenhaushygiene, Klinikum rechts der Isar, Technische Universität München, München, Deutschland; ${ }^{3}$ Fakultät für Medizin, Institut für Virologie, Klinikum rechts der Isar, Technische Universität München, München, Deutschland; ${ }^{4}$ Fakultät für Medizin, Stabsstelle für Qualitäts- und Risikomanagement, Klinikum rechts der Isar, Technische Universität München, München, Deutschland; ${ }^{5}$ Fakultät für Medizin, Unternehmenskommunikation, Klinikum rechts der Isar, Technische Universität München, München, Deutschland; ${ }^{6}$ Fakultät für Medizin, Pflegedirektion, Klinikum rechts der Isar, Technische Universität München, München, Deutschland;

${ }^{7}$ Fakultät für Medizin, Institut für medizinische Mikrobiologie, Immunologie und Hygiene, Klinikum rechts der Isar, Technische Universität München, München, Deutschland; ${ }^{8}$ Fakultät für Medizin,

Krankenhausapotheke, Klinikum rechts der Isar, Technische Universität München, München, Deutschland ${ }^{9}$ Fakultät für Medizin, Institut für diagnostische und interventionelle Radiologie, Klinikum rechts der Isar, Technische Universität München, München, Deutschland; ${ }^{10}$ Fakultät für Medizin, Klinik und Poliklinik für Innere Medizin II, Klinikum rechts der Isar, Technische Universität München, München, Deutschland; "Fakultät für Medizin, Klinik und Poliklinik für Innere Medizin II (Infektiologie), Klinikum rechts der Isar, Technische Universität München, München, Deutschland

\title{
Strategie einer universitären Notaufnahme für das COVID-19- Management im Rahmen einer beginnenden Epidemie
}

\section{Einführung}

Die Weltgesundheitsorganisation (WHO) hat am 30.01.2020 eine "gesundheitliche Notlage von internationaler Tragweite“ aufgrund der stark zunehmenden Fälle an Erkrankungen mit dem neuartigen Coronavirus SARS-CoV-2 ausgerufen [1]. Die durch das Virus ausgelöste fieberhafte Atemwegserkrankung „coronavirus disease 2019“ (COVID-19) wurde zu diesem Zeitpunkt vor allem in China und hier insbesondere in der $\mathrm{Hu}$ bei-Provinz um die Metropole Wuhan nachgewiesen [2]. Etwa einen Monat später, gegen Ende Februar, stiegen die Fallzahlen der COVID-19-Erkrankungen auch sprunghaft in Europa, explizit in Norditalien und auch in Deutschland, an. Zuvor war es im Januar nur zu einem einzelnen Eintrag in die Region München durch eine chinesische Mitarbeiterin gekommen [3]. Grundsätzlich stufte die nationale Seuchenschutzbehör- de, das Robert Koch-Institut, das Risiko bezüglich des Auftretens einer Pandemie Ende Februar noch als "mäßig“ ein. Am 17.03.2020 wurde das Risiko neu bewertet und auf „hoch“ klassifiziert [4]. Am 11.03.2020 wurde von der WHO die Ausbreitung von COVID-19 als Pandemie ausgerufen [1].

Als Inkubationszeit wurden bis $\mathrm{zu}$ 14 Tage ermittelt, wobei die Übertragung mehrheitlich durch Tröpfchen-, Schmierinfektion aus dem Respirationstrakt erfolgt. Die Möglichkeit einer fäkal-oralen Übertragung wird diskutiert, ist aber nicht abschließend geklärt [5]. Ein hohes Risiko einer Übertragung besteht daher vor allem für Menschen, die Kontakt zu Infiziernten hatten der länger als $15 \mathrm{~min}$ andauerte und die räumliche Distanz wengier als 2 Meter betrug. Ob die Infektion auch bei asymptomatischen Trägern möglich ist, wird kontrovers diskutiert $[3,6]$.
Zur Diagnostik ist ein Erregerdirektnachweis mittels PCR-Test aus Abstrichen des oberen Respirationstrakts, aus dem Sputum oder aus der bronchoalveolären Lavageflüssigkeit geeignet [7]. Radiologische Bildgebung kann zusammen mit der virologischen Diagnostik, laborchemischen Indikatoren wie der Leukopenie sowie der Klinik zur Verbesserung der Diagnostik beitragen [8, 9], während die Serologie in der Akutdiagnostik eine untergeordnete Rolle spielt [10]. So waren SARS-CoV-2-Antikörper bei $50 \%$ hospitalisierter Patienten nach 7 Tagen und bei $100 \%$ nach 14 Tagen nachweisbar [11].

Wie auch schon vorangegangene Pandemien gezeigt haben, sind in dieser Notlage insbesondere die Notfallstrukturen des Gesundheitssystems aller Bereiche gefordert. Dies betrifft den öffentlichen Gesundheitsdienst, die ambulante und stationäre Versorgung sowie die Infrastruktur im Gesundheitswesen und dem 

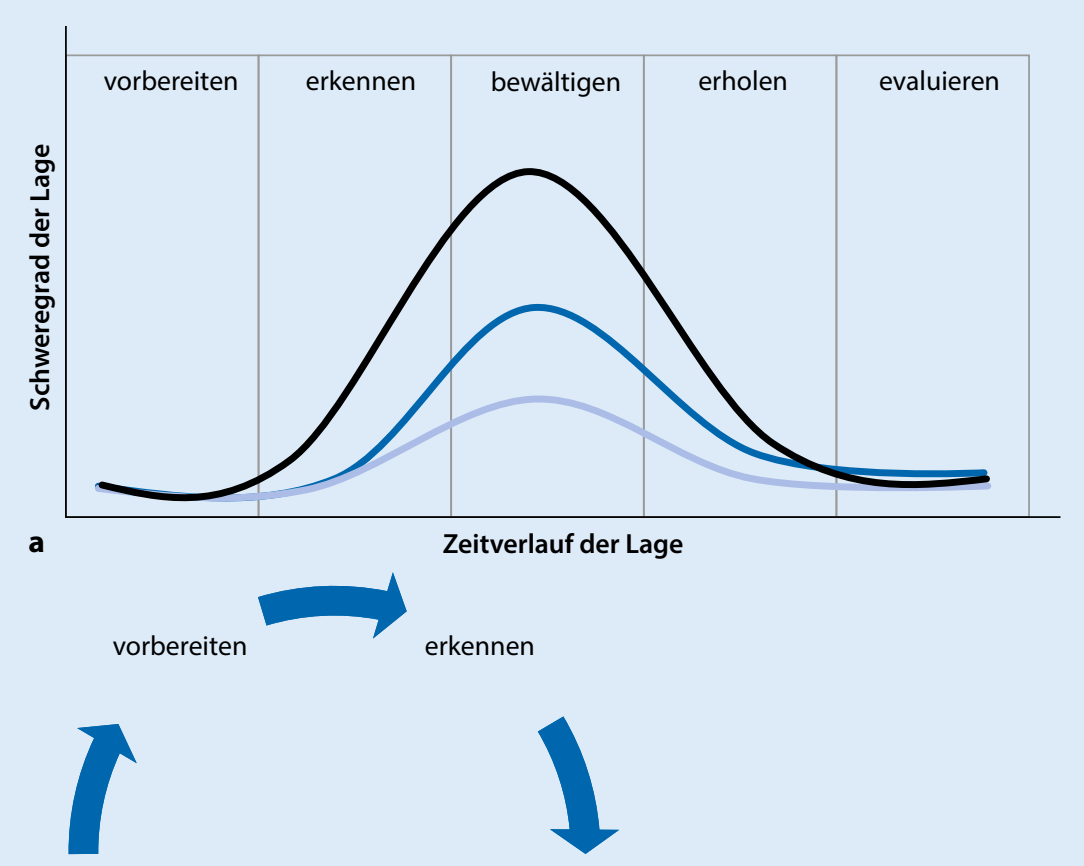

evaluieren bewältigen

b

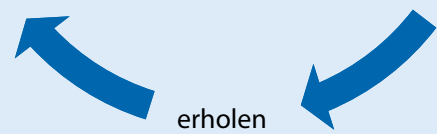

Abb. $1 \Delta$ a Ausmaß und Verlauf von epidemisch bedeutsamen Lagen (schwarze Linie: hoher Schweregrad, dunkelblaue Linie: mittlerer Schweregrad, hellblaue Linie: geringer Schweregrad der Lage); b Zyklus Krisenmanagement. (Modifiziert nach [15])

öffentlichen Leben gleichermaßen. Vorhandene Notfallpläne, einschließlich der Pandemiepläne, wurden aufgrund der fehlenden Notwendigkeit häufig seit Jahren nicht mehr geprüft und aktualisiert. Schließlich liegt die letzte Pandemie, die „Asiengrippe im Jahre $1957^{\text {“ lange }}$ zurück [12].

Die ungeplante Vorbereitung und der Übergang von Routineversorgung in Pandemieversorgung stellen insbesondere Kliniken und ihre Notaufnahmen vor große Herausforderungen. Dieser Artikel soll veranschaulichen, wie sich insbesondere Notaufnahmen auf das Management einer Pandemie, hier am Beispiel COVID-19, konkret und praxisnah vorbereiten können, was am Beispiel der Einrichtung des Klinikums rechts der Isar der Technischen Universität München erläutert werden soll.

\section{Rahmenbedingungen}

\section{Klinikum rechts der Isar der TU München}

Das Klinikum rechts der Isar der Technischen Universität München ist gemäß $₫ 136 c$ Absatz 4 Sozialgesetzbuch (SGB) V ein Krankenhaus der „umfassenden Notfallversorgung - Stufe 3“. Es verfügt über eine zentrale Notaufnahme (ZNA) mit Aufnahmestation, Intensivkapazität, entsprechend geforderte Fachabteilungen (innere Medizin, Chirurgie inklusive Unfall-, Neuro- und Viszeralchirurgie, Neurologie, Neuroradiologie, Anästhesie und andere) sowie einen Hubschrauberlandeplatz. Zur Schlaganfall- und Herzinfarktversorgung werden eine Stroke-Unit und Chest-Pain-Unit vorgehalten.

Zudem ist das Klinikum als überregionales Traumazentrum zertifiziert und erfüllt gemäß dem Schwerstverletzungsartenverfahren (SAV) die höchste Stufe bei der Versorgung von Verletzungen (\$ 34 SGB VII).

\section{Zentrale Notaufnahme}

Die zentrale Notaufnahme wurde durch einen Zusammenschluss einer konservativen Notaufnahme (hauptsächlich innere Medizin und Neurologie) und einer chirurgischen Notaufnahme erst im Vorjahr räumlich und organisatorisch zusammengeführt. Notwendige Umbauarbeiten erfolgen im laufenden Betrieb und ziehen teilweise Betriebseinschränkungen nach sich. Die ZNA verfügt über einen voll funktionsfähigen Schockraumbereich mit angegliederter Computertomographie, wobei die Schockraumerstversorgung in einem interdisziplinären Team aus Chirurgie, innerer Medizin, Neurologie, Anästhesie und Radiologie stattfindet.

Grundsätzlich ist die medizinische Ersteinschätzung der Notfallpatienten, die mit dem Rettungsdienst eingewiesen werden, und jener, die sich selbstständig vorstellen, mittels des in Deutschland etablierten Manchester-Triage-Systems (MTS) organisiert [13]. Die Triage erfolgt nach Identifikation des Patienten im eingesetzten Softwareapplikationsverfahren und die Patienten werden nach Dringlichkeit der Behandlung zugeführt.

\section{Pandemiepläne}

Vorhandene Pandemiepläne, wie beispielsweise der Influenzapandemieplan des Robert Koch-Instituts [14], haben das übergeordnete Ziel, die Morbidität und Mortalität zu reduzieren, die Versorgung Erkrankter sicherzustellen und das öffentliche Leben mit seinen essenziellen Teilen aufrechtzuerhalten. Hierbei werden in der Regel mindestens drei Stufen vorgesehen: 1. Eindämmung („,containment"), 2. Schutz von Menschen mit Risiko (,protection") und 3. Minderung der Folgen bei nicht aufhaltbarer Erregerverbreitung („mitigation“): Ziel der ersten Stufe, der Eindämmungsstrategie, ist es, Infizierte sehr früh zu identifizieren, Kontaktpersonen lückenlos nachzuverfolgen und 
damit die Verbreitung wirksam zu unterbinden oder zumindest erheblich zu verlangsamen. Ziel der zweiten Stufe, ist es, besonders gefährdete Risikogruppen bei fortgesetzter Übertragung vor den Folgen der Infektion zu bewahren, und Ziel der dritten Stufe ist es, Überlastungen der Versorgungssysteme zu vermeiden und die Verringerung negativer Auswirkungen auf die Gesellschaft und das soziale Leben. Hierbei existieren neben den nationalen auch regionale Pandemiepläne der Länder sowie teilweise auch einzelner Einrichtungen der Gesundheitsversorgung. Am Klinikum rechts der Isar wurde im letzten Jahr der Katastrophenplan um das Kapitel „Infektiöse Gefahrenlagen“ erweitert. Auf diesen Plan könnte nun im Rahmen der COVID-19-Pandemie teilweise zurückgegriffen werden.

\section{Lageanalyse und COVID-19- spezifische Maßnahmen}

Vor Beginn aller Maßnahmen ist die Identifikation des Risikoszenarios erforderlich: Obgleich Anfang Januar die COVID-19-Infektionen in Hubei noch weit weg vom deutschen Alltag schienen, ergab sich bereits ein deutlich erkennbares Risiko. Fachexperten des Klinikums analysierten die Situation und bewerteten sie bereits Mitte Januar als epidemiologisch relevant [15]. Diese Erkenntnis ist nach der grundsätzlichen Vorbereitung der initiierende Schritt (• Abb. 1a) weiterer Maßnahmen. Im folgenden Teil sollen die weiteren Schritte dargelegt werden.

\section{Maßnahmen}

\section{Organisation}

Um eine effektive Analyse, Vorbereitung, Bewertung, Bewältigung, Exekution und Evaluation aller erforderlichen Maßnahmen am Klinikum zu steuern, wurde ein Expertenteam mit beteiligten Fachberatern aus den Bereichen Infektiologie, Virologie, Notfallmedizin, Unternehmenskommunikation, Pflegedirektion und Hygiene ad hoc durch den Klinikumsvorstand eingesetzt.

Notfall Rettungsmed 2020 - 23:578-586 https://doi.org/10.1007/s10049-020-00759-8

(c) Springer Medizin Verlag GmbH, ein Teil von Springer Nature 2020

M. Dommasch · F. Gebhardt - U. Protzer · A. Werner · E. Schuster · C. Brakemeier · J. Mayer · S. Feihl · C. Querbach · R. Braren · M. Treiber · F. Geisler · C. D. Spinner

\section{Strategie einer universitären Notaufnahme für das COVID-19- Management im Rahmen einer beginnenden Epidemie}

Zusammenfassung

Durch die weltweit steigenden Zahlen an "Coronavirus disease 2019"(COVID-19)Infektionen besteht für sämtliche Kliniken die Aufgabe, sich der Herausforderung einer Pandemie zu stellen. Es gilt insbesondere auch für die Notaufnahmen, sich auf vollständig veränderte Arbeitsabläufe vorzubereiten und sie umzusetzen. Dies betrifft insbesondere den Bereich Patientenscreening und -selektion (Triage). Auch mit anderen Fachbereichen wie der Hygiene, Infektiologie oder Virologie muss Hand in Hand zusammengearbeitet werden, um vor, während und nach Abschluss der Diagnostik entsprechende
Behandlungskonzepte zu realisieren. Darüber hinaus sind Kommunikation und Qualitätsund Risikomanagement nebst den klinischen Bereichen von hoher Relevanz. Dieser Artikel beschreibt an einem Beispiel, wie sich Notaufnahmen hier am Beispiel COVID19 (coronavirus disease 2019) konkret und praxisnah auf eine Pandemie vorbereiten können.

Schlüsselwörter

Notfallzentrum · Triage · SARS-CoV-2 .

Coronavirus · Pandemie

\section{Strategy for university emergency room management at the beginning of an epidemic using COVID-19 as an example}

\section{Abstract}

Due to the increasing number of COVID19 infections worldwide, all hospitals are faced with the challenge associated with the pandemic. In particular, emergency rooms must prepare and implement completely new workflows. This applies in particular to patient screening and selection (triage). Close cooperation with other specialist areas such as hygiene, infectiology or virology is also necessary in order to implement appropriate treatment concepts before, during and after the diagnosis is completed In addition, communication and quality and risk management are highly relevant in addition to the clinical aspects. This article uses COVID-19 as an example to describe how emergency rooms can prepare for a pandemic.

Keywords

Emergency center - Triage - SARS-CoV-2 .

Coronavirus - Pandemic
Das Expertenteam berät hierbei den Vorstand als zentrale Klinikumleitung in allen zugehörigen Angelegenheiten. Ziel ist es, die Betriebsfähigkeit des Klinikums, einschließlich sämtlicher assoziierter Prozesse, so weit wie möglich so anzupassen, dass die Mitwirkung in der Krankenversorgung nicht gefährdet wird. Bedarfsweise werden hierzu alle verfügbaren Fachabteilungen des Klinikums involviert. In Anlehnung an den allgemeinen Krisenmanagementzyklus [15] agiert das Expertenteam und reagiert auf die aktuelle Lage (• Abb. 1b).

\section{Risikomanagement}

Grundsätzlich gilt, dass der Vorstand des Klinikums bei akuten Risiken und besonderen Ereignissen stets vollständig und zeitnah $\mathrm{zu}$ informieren ist. Hier$\mathrm{zu}$ wird der Vorstand täglich in einem informativen Jour fixe am Mittag (Briefing mit Lageanalyse, Festlegung von Maßnahmen; exemplarischer Tagesplan in - Tab. 1) beraten und beteiligt nach Entscheidung zur Exekution die entsprechenden Katastrophenschutzstabstellen aus dem Katastrophenschutzplan (S1: Personal, S2: Lage, S3: Maßnahmen, S4: Versorgung, S5: Presse- und Öffentlichkeitsarbeit, S6: Informationstechnologie). Etablierte und sichere Prozesse/ 
Tab. 1 Beispielhafter Tagesplan des Expertenteams COVID-19 am Klinikum rechts der Isar

\begin{tabular}{|c|c|c|}
\hline Zeit & Aufgabe & Inhalt \\
\hline \multirow[t]{2}{*}{ 08:00-09:00 } & \multirow{2}{*}{$\begin{array}{l}\text { Organisation/ } \\
\text { Regelarbeit }\end{array}$} & Update zum Vortag \\
\hline & & Überblick verschaffen \\
\hline 09:00-10:00 & Meeting & Prozesse neu definieren oder an die aktuelle Lage anpassen \\
\hline \multirow[t]{2}{*}{$10: 00-12: 45$} & \multirow{2}{*}{$\begin{array}{l}\text { Organisation/ } \\
\text { Regelarbeit }\end{array}$} & Neuerungen im Prozess zur Umsetzung vorbereiten \\
\hline & & $\begin{array}{l}\text { Kommunikation mit anderen Beteiligten sowohl nach in- } \\
\text { tern (Bereichsleiter, Stabsstellen, Administration etc.) als } \\
\text { auch nach extern (Kliniken, Behörden) }\end{array}$ \\
\hline $12: 45-13: 00$ & $\begin{array}{l}\text { Vorstands- } \\
\text { abstimmung }\end{array}$ & $\begin{array}{l}\text { Beschluss der Vorgehensweise und Neuerungen mit dem } \\
\text { Vorstand }\end{array}$ \\
\hline \multirow[t]{3}{*}{$13: 00-13: 30$} & \multirow{3}{*}{$\begin{array}{l}\text { Stabsstellen- } \\
\text { besprechung }\end{array}$} & Anweisung der Beschlüsse an die Stabsstellen \\
\hline & & Abfrage der einzelnen Stabsstellen \\
\hline & & Informationsaustausch \\
\hline \multirow{2}{*}{$\begin{array}{l}\text { 13:30-Dienst- } \\
\text { ende }\end{array}$} & \multirow{2}{*}{$\begin{array}{l}\text { Organisation/ } \\
\text { Regelarbeit }\end{array}$} & Umsetzen der Vorgehensweise \\
\hline & & Anweisung der Mitarbeit über Neuerungen im Prozess \\
\hline \multirow{2}{*}{$\begin{array}{l}\text { Dienstzeiten } \\
\text { und } \\
\text { Wochenende }\end{array}$} & \multirow[t]{2}{*}{ Rufbereitschaft } & Kontrolle der Lage innerhalb der Klinik \\
\hline & & $\begin{array}{l}\text { Kommunikation ins Expertenteam bei Störung oder Fehlern } \\
\text { in den definierten Prozessen }\end{array}$ \\
\hline
\end{tabular}

Abläufe und Strukturen im Klinikum werden hierbei genutzt und ggf. angepasst.

So werden bedarfsweise die Hygienemaßnahmen, wie das Tragen des MundNasen-Schutzes oder die Essensausgabe in der Mensa, Umbaumaßnahmen zur räumlichen Trennung der Patienten oder Aufstockung der Intensivkapazitäten diskutiert und an die Gegebenheiten angepasst. Hier ist der Input aller Disziplinen des Expertenteams von Bedeutung. Die Maßnahmen müssen nach Kosten-Nutzen kalkuliert, ausgearbeitet, kommuniziert und exekutiert werden.

\section{Kommunikation}

Eine besondere Herausforderung stellt die Kommunikation der Risiko- und Krisensituation dar. Eine rasche, umfassende und konsistente Information aller Akteure unter Nutzung der verfügbaren Medien ist zu organisieren.

Sitzungen oder Besprechungen können nur unter Einhaltung der Hygienemaßnahmen abgehalten werden. Daher sollten diese auf ein Mindestmaß reduziert werden bzw. sollte auf digitale Möglichkeiten, wie z.B. Videokonferenzen, zurückgegriffen werden.

Transparenz ist oberstes Gebot, um Entscheidungen zum Schutzverhalten, zu Änderungen betrieblicher Abläufe und zu Maßnahmen für alle Beteiligten, für alle Mitarbeiter und für vor- und nachgeordnete Institutionen nachvollziehbar zu vermitteln. Trotz vorhandener Standards und Abläufe stellt sich in zeitlicher schneller Abfolge ein Veränderungsprozess mit hoher Komplexität ein, der im gesamten Team des Klinikums bewältigt werden muss. Die Kommunikationswege müssen der jeweils aktuellen Lage angepasst werden. Dabei sind sowohl einfache Informationswege für größere Personengruppen als auch kleinste Dienstwege bei der internen Kommunikation zu gestalten. Die Akzeptanz, das Verantwortungsbewusstsein („awareness“) und die kooperative Mitarbeit aller Beteiligten hängen direkt davon $\mathrm{ab}$, wie stark sich diese abgeholt, integriert und wertgeschätzt fühlen.

Generell gilt für die Kommunikationsstrategie, nicht nur Informationen zu vermitteln, sondern diese auch auf Dialog und Rückmeldungen auszulegen. Es wurde eine strukturierte Informationsvermittlung mit einer effektiven Koordinierung aufgebaut, wobei E-Mail, Intranet, Mitarbeitertelefon, Kommunikation über Dienstvorgesetzte und Kommunikation auf öffentlichen Plattformen (beispielsweise Schulungsvideos) konsequent genutzt wurden. Trotz dieser breiten Kommunikationswege ist eine Übermittlung neuer Informationen an eine breite Masse von Mitarbeitern schwierig und nie zu $100 \%$ umzusetzen. Insbesondere E-Mail und Intranet scheinen gegenüber dem Mitarbeitertelefon nur gering genutzt zu werden. Im Verlauf der Pandemie musste insbesondere das Mitarbeitertelefon massiv ausgebaut werden, um unter anderem auch direkt und indirekt betroffene Mitarbeiter zu unterstützen.

Ziel der Ausweitung der Kommunikation ist es, die Mitarbeiter über alle Abläufe im Klinikum und auch Anpassungen im Rahmen der Pandemie transparent zu informieren. Hierzu zählen jegliche Informationen, die der Sicherheit der Mitarbeiter dienen, aber auch allgemeine Punkte, wie der Verbrauch an Schutzkleidung, wie der Nachschub geregelt ist, wie die Patientenzahlen sich entwickeln etc.

\section{Patientenversorgung}

Nachdem im Vorliegenden COVID-19Szenario die fieberhafte Atemwegsinfektion mit Aerosolübertragung als wesentliches Risiko identifiziert wurde, müssen Menschen mit entsprechend fieberhafter Atemwegsinfektion zum frühestmöglichen Zeitpunkt vom Besucherstrom der Notaufnahme nach Betreten des Klinikums getrennt werden, ggf. sind alle $\mathrm{Be}$ sucher/Patienten, die das Klinikum betreten, zu screenen.

Dies kann zum einen durch generelle Temperaturkontrollen erfolgen. Hierbei sollte aufInfrarotthermometer zurückgegriffen werden, da diese kontaktlos angewendet werden können. Von Nachteil ist jedoch eine hohe Messvarianz und damit verbunden eine Ungenauigkeit dieser Methode.

Weiter sollte das Infektionsrisiko mittels dezidierter Fragen nach Symptomen (Husten, Atemnot, Fieber, Geschmacksund Geruchsverlust), Kontakt zu infizierten Mitmenschen, abgelaufener Infektion/Erkrankung und stattgehabter Testung eingegrenzt werden.

\section{Identifikation von Verdachtsfällen in der ZNA}

Erster Anlaufpunkt aller Notfallpatienten ist der Ort der Ersteinschätzung (Triagestelle). Hier findet im Regelbetrieb eine Selektion nach Schweregrad der Erkran- 
Tab. 2 Patientenselektion und COVID-19-Screening in Abhängigkeit der Pandemiestufe

\begin{tabular}{|c|c|c|}
\hline $\begin{array}{l}\text { Pandemie- } \\
\text { stufe }\end{array}$ & Patientenselektion & COVID-19-Screening \\
\hline \multirow[t]{3}{*}{ Frühe Phase } & Kontaktlos & \multirow[t]{3}{*}{ Alleinig mittels PCR } \\
\hline & Wartemarkenautomat & \\
\hline & Separater Wartebereich & \\
\hline \multirow{4}{*}{$\begin{array}{l}\text { Spätere } \\
\text { Phase }\end{array}$} & Früher Kontakt vor der ZNA & Algorithmus \\
\hline & Temperarturmessungen & $\begin{array}{l}\text { Computertomographie als wichtigstes Diagnosti- } \\
\text { kum mit dem Vorteil eines schnellen Ergebnisses }\end{array}$ \\
\hline & Separater Wartebereich & \multirow[t]{2}{*}{ PCR nur zur endgültigen Sicherung } \\
\hline & Separate Behandlung & \\
\hline
\end{tabular}

kung statt. Im Rahmen der sich häufenden COVID-19-Erkrankungen ist jedoch nicht nur der Schweregrad der zugrunde liegenden Erkrankung von Bedeutung, sondern auch der potenzielle Infektionsstatus im Hinblick auf die Transmission.

Der Bereich der Triage ist gerade bei hohem Patientenaufkommen schwer geeignet, um eine solche Selektion nach Infektionsstatus durchzuführen, da insbesondere hier das Potenzial der Verbreitung einer aerosolübertragbaren Erkrankung aufgrund der baulichen und strukturellen Begebenheiten am höchsten ist - höchster Patientendurchsatz auf begrenzter Fläche mit nur geringer Frischluftzufuhr.

Ziel muss es daher sein, die potenziell infektiösen Patienten frühzeitig zu identifizieren und zu isolieren, bestenfalls noch vor Betreten eines vulnerablen Bereichs wie der Notaufnahme. Patienten, die über den Rettungsdienst im Krankenhaus vorgestellt werden, sind eher weniger problematisch zu werten, da entweder eine telefonische Voranmeldung erfolgt oder schon bei der Disposition ein entsprechender Vermerk hinterlegt wurde und die Patienten ausreichend mit Schutzkleidung versorgt wurden.

Herausforderungsvoller sind Notfallpatienten, die sich selbstständig (d.h. eigeninitiativ oder mittels Einweisung über den Haus- oder KVB-Arzt) in der ZNA vorstellen. Insbesondere diese Patienten sollten bei einem Verdacht auf eine SARS-CoV-2-Infektion, d.h. bei Symptomen eines fieberhaften Atemwegsinfekts, von anderen Patienten schnellstmöglich separiert werden. Neben dem Schutz der Mitpatienten ist natürlich auch der Schutz der Mitarbeiter nicht unerheblich.
Diese frühzeitige Selektion von Patienten kann anhand des Patientenaufkommens in verschiedenen Stufen ablaufen (• Tab. 2).

So zeigte sich, dass bei noch wenigen potenziell infektiösen Patienten ein Wartemarkenautomat und eine Beschilderung in einen zusätzlichen Wartebereich einfach und ohne Mitarbeiterkontakt zu einer Separation von Patienten beitragen können. Die Wartemarkenausgabe muss hierbei im Bereich vor der Triage bzw. ZNA unter (Video-)Sichtkontrolle installiert und eine entsprechende Beschilderung zu einem separaten Wartebereich etabliert werden. Weite Wege sollten für den Patienten und auch das Personal vermieden werden, um zum einen eine schnelle Versorgung trotz dieses „Umwegs" zu gewährleisten, aber auch um eine weitere Ausbreitung so gering wie möglich zu halten. Die Mitarbeiter der ZNA erhalten über einen Aufrufmonitor die Information, dass ein Patient sich im separaten Wartebereich befindet, und können die Ersteinschätzung und Behandlung des potenziell infektiösen Patienten geordnet vorbereiten (persönliche Schutzausrüstung [PSA] etc.).

Bei zunehmender Patientenzahl ist dieses Vorgehen nicht mehr sinnvoll und eine Selektion der Patienten wird im Falle der fieberhaften COVID-19-Erkrankung mittels Temperaturmessung vor der Notaufnahme durchgeführt. Patienten mit erhöhter Temperatur und/ oder Atemwegssymptomen werden strikt von den übrigen Patienten getrennt und nach einem eigenen Algorithmus auf COVID-19 gescreent.

\section{COVID-19-Triage mittels Algorithmus}

Zhang et al. entwickelten einen Algorithmus anhand ihrer Erfahrungen mit COVID-19-Patienten in Wuhan, der in modifizierter Form an unserem Haus Anwendung findet (• Abb. 2; $[9,16])$. In der initialen Phase der COVID-19-Erkrankung zeigen Patienten häufig unspezifische Symptome: Schüttelfrost und/oder Atemwegssymptome. Eine schnelle Identifikation von Verdachtspatienten ist daher sehr schwer und bedarf neben der Klinik weiterer diagnostischer Parameter. Im angewendeten Algorithmus sind die wichtigsten Parameter die Computertomographie des Thorax, Laborwerte (Lymphozytopenie) und die Körpertemperatur. Auf Basis dessen kann rasch eine klinische Entscheidung zur weiteren COVID-19-Diagnostik mittels PCRTest, empirischen antimikrobiellen Therapie sowie Isolationsempfehlungen abgeleitet werden. Die Low-dose-Computertomographie des Thorax erfasst zuverlässig charakteristische fleckige bipulmonale Infiltrationen, die zu großen Milchglastrübungen fortschreiten können, und ist im Vergleich zum konservativen Thoraxröntgenbild in ihrer Sensitivität deutlich höher. Neben dem diagnostischen Entscheidungsbaum umfasst dieser Algorithmus auch therapeutische Ansätze, die dann je nach Schweregrad der Erkrankung im weiteren stationären oder ambulanten Setting durchgeführtwerden können.

\section{Management von Verdachtsfällen in der ZNA}

Sollte eine stationären Behandlung nötig sein, ist eine geeignete Isolation dieser Verdachtsfälle zu organisieren. Hierfür kann beispielsweise die assoziierte Aufnahmestation genutzt werden, sodass hier ein Hybridbereich entsteht mit einem gemischten Anteil an Verdachtsfällen und Patienten, die aus anderen Gründen die Notaufnahme in Anspruch genommen haben. Die Aufnahmestation ist räumlich in der Nähe und wird von den Mitarbeitern der ZNA betreut, die in alle Handlungsabläufe eingewiesen sind und Hand in Hand mit der ZNA arbeiten. Bestenfalls erfolgt eine Trennung des Personals in Mitarbeiter, die nur Verdachts- 


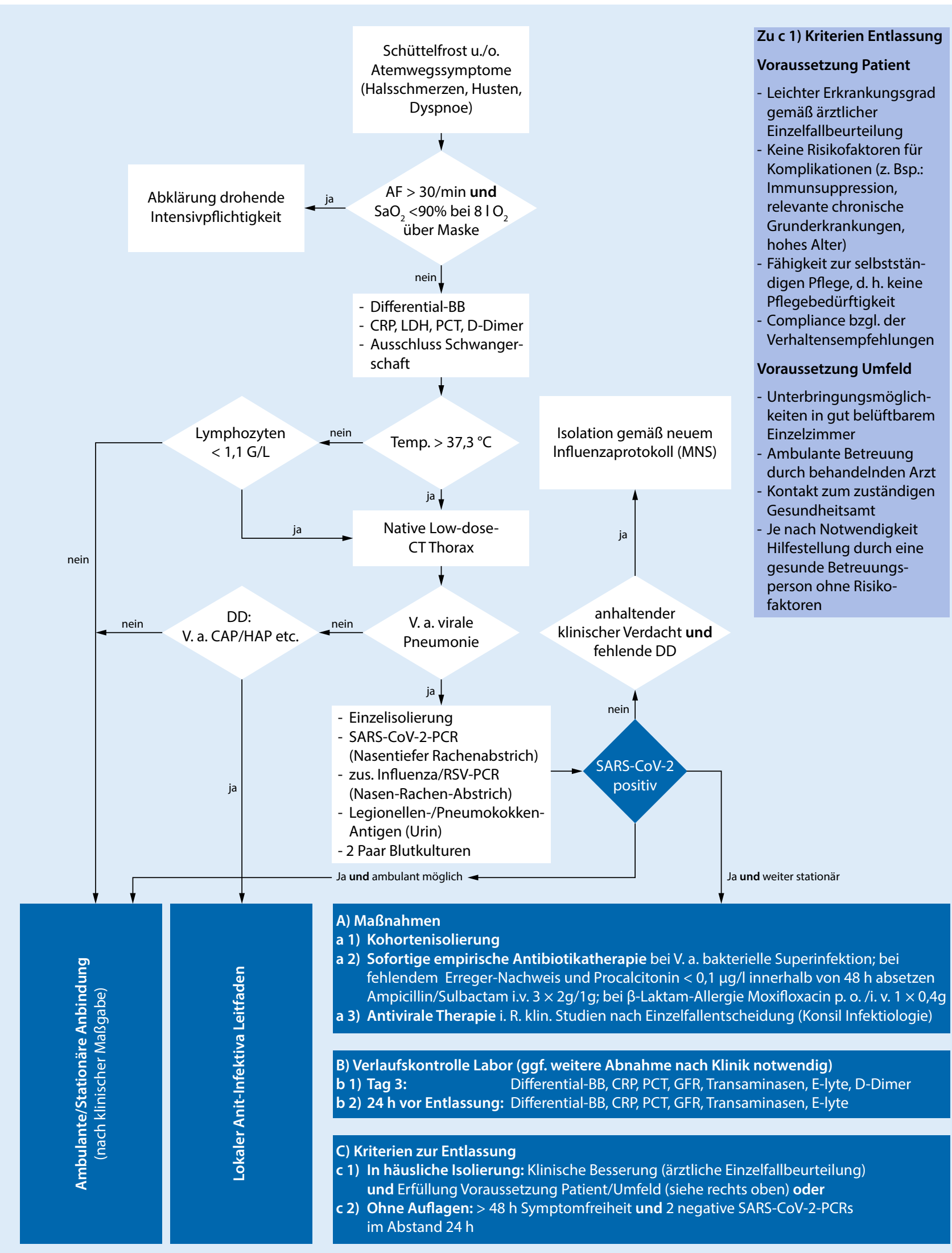

Abb. 2 \ Diagnostik- und Therapiealgorithmus bei V.a. COVID-19. (Modifiziert nach [9]) 


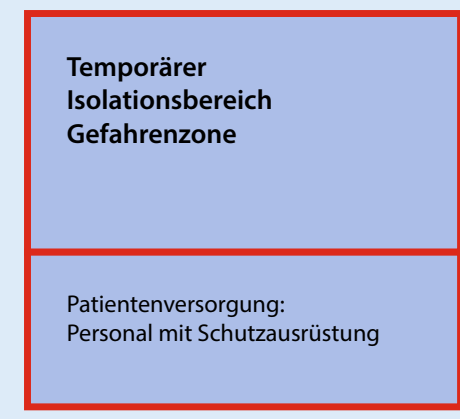

Dekontaminationsbereich

Entkleiden bis auf Fußbekleidung: Auf ausreichende Größe der Fläche achten

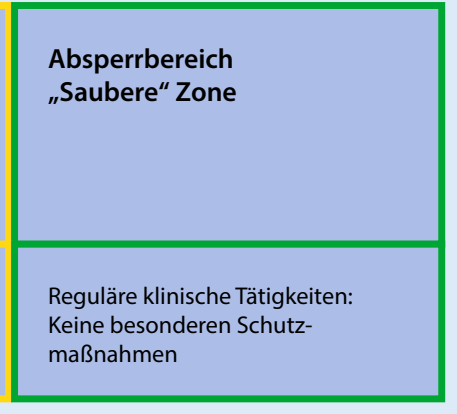

Abb. $3<$ Übersicht der verschiedenen Gefahrenbereiche bei der Versorgung von infektiologischen Patienten fälle behandeln, und Mitarbeiter, die die übrigen Notfallpatienten betreuen.

Die Patienten mit Verdacht auf COVID-19 werden hier isoliert, bis die diagnostische Klärung des Falls abgeschlossen ist. Dies kann bei nicht eindeutigen Befunden (CT positiv, Abstrich negativ) jedoch einige Stunden, ggf. Tage in Anspruch nehmen. So werden alle unklaren Befunde durch einen zweiten Abstrich nach $24 \mathrm{~h}$ reevaluiert.

\section{Umgang mit bestätigten Fällen}

Um die Betriebsfähigkeit der ZNA zu erhalten, ist die zeitnahe Verlegung der Patienten auf die weiterversorgenden Stationen entscheidend: Bei negativer SARS-CoV-2-Diagnostik und klinisch fehlenden Verdachtsmomenten für eine COVID-19-Erkrankung erfolgt die Weiterbehandlung in peripheren Bettenstationen, während die Aufnahme von bestätigten Fällen in Isolierstationen erfolgt. Hierfür werden einzelne Stationen in ihrer Funktion umgewidmet und einzig der Behandlung von COVID-19Patienten zur Verfügung gestellt. Der Transport ist hierbei nach den Hygieneempfehlungen auf dem kürzesten Weg in die Versorgungsbereiche durchzuführen.

Aufgrund der zu erwartenden hohen Anteile an Patienten, die eine intensivmedizinische Betreuung, auch über einen längeren Zeitraum, benötigen, ist ein rechtzeitiges Aktivieren aller bereitstehenden Ressourcen in diesem Bereich nötig. Auch mit dem Hintergedanken, dass es nicht zu einem "Exit-Block“ im Sinne fehlender Abverlegungsoptionen von infektiösen Intensivpatienten in der Notaufnahme kommen darf, sind entsprechende gestufte Pläne notwendig.
Während des gesamten Workflows wird zwischen dem temporären Gefahrenbereich (Versorgungsbereich infizierter Patienten), dem Dekontaminationsbereich (Schleuse und/oder Vorplatz vor Patientenzimmer) und dem Absperrbereich (saubere Zone) unterschieden (- Abb. 3). Diese Unterteilung ist insbesondere vor dem Hintergrund der benötigten persönlichen Schutzausrüstung (PSA) des Personals entscheidend.

\section{Infektionspräventive Maßnahmen, persönliche Schutzausrüstung (PSA)}

Die Auswahl der infektionspräventiven Maßnahmen orientiert sich an den Übertragungswegen der Infektionserkrankung, hier Übertragung über Tröpfchen bzw. Aerosole sowie Handkontakte. Durch den Ausschuss für Biologische Arbeitsstoffe (ABAS) wurde SARS-CoV-2 vorläufig in die Risikogruppe 3 nach Biostoffverordnung eingestuft [17].

Die PSA besteht aus flüssigkeitsdichtem Schutzkittel, Einmalhandschuhen, dicht anliegender FFP2-Atemschutzmaske (FFP3 bei ausgeprägter Aerosolexposition, z. B. Intubation) und Schutzbrille. Aus Praktikabilitätsgründen wird ein doppeltes Handschuhpaar getragen, was das kontaminationsfreie Ausziehen der PSA erleichtert.

Idealerweise erfolgt zunächst eine Isolierung der Patienten, idealerweise in einem Isolierzimmer mit Schleuse oder Vorraum. Eine ausreichende Größe der Schleuse erlaubt eine organisatorische Trennung von reiner und unreiner Seite, muss aber ggf. bei fehlenden baulichen Voraussetzungen alternativ ersetzt werden. Das An- und Ablegen der PSA sowie die Entsorgung mit Zwischenlagerung kontaminierter Wäsche oder Abfälle wird durch Schleusen erleichtert.

Aufgrund der bevorstehenden Umbauten stehen am MRI keine Schleusen zur Verfügung. Die Schutzkleidung wird deshalb in Hygienezentren vor den Isolierzimmern gelagert und vor dem Zimmer angelegt. Nach der jeweiligen Tätigkeit wird die PSA im Zimmer kontaminationsfrei abgelegt, zunächst Handschuhe, Schutzkittel, dann nach Händedesinfektion Brille und Maske, und in einem geschlossenen Behälter verworfen.

Aufgrund fehlender raumlufttechnischer Anlagen wird großer Wert auf eine ausreichende Belüftung gelegt. Wenn möglich wird während der Tätigkeit im Zimmer das Fenster geöffnet. Weiterhin soll der Patient sofern toleriert so lange einen Mund-Nasen-Schutz tragen.

Entscheidend für das korrekte Anwie das kontaminationsfreie Ablegen der PSA und die Vermeidung von Sicherheits- und Hygienelücken ist ein sicherer Umgang der Mitarbeiter mit der PSA. Ein intensives Schulen ist hierfür Voraussetzung. Insbesondere in der Vorbereitungsphase sowie begleitend bei der Anwendung im Rahmen der Patientenversorgung ist eine engmaschige Begleitung notwendig.

Neben den ärztlichen und pflegerischen Mitarbeitern ist dabei auch das versorgende Personal wie Reinigungskräfte, Physiotherapeuten etc. einzubeziehen.

Um die Mitarbeiter und die Patienten bestmöglich zu schützen, wurde im Verlauf der Pandemie dazu übergegangen, allen anwesenden Personen im Klinikum einen MNS auszuhändigen. Zudem wurden die Eingänge ins Klinikum reduziert und überwacht. Hygieneinformationen 
wurden online und durch entsprechende Beschilderung im Klinikum allen Beteiligten aufgezeigt.

\section{Aufrechterhaltung der Versorgung}

Wesentlich für den ausreichenden Arbeitsschutz und damit auch die Versorgung entsprechender Patienten ist die ausreichende Verfügbarkeit von persönlicher Schutzausrüstung sowie Händeund Flächendesinfektionsmitteln.

Zur Basisbevorratung besteht dazu für Krankenhaus-, krankenhausversorgende und öffentliche Apotheken eine gesetzliche Bevorratungspflicht ( $\$ 73$ AMVSG) im Umfang des durchschnittlichen Bedarfs von zwei bzw. einer Woche.

Je nach Entwicklung einer Lage kann dieser Bestand in kurzer Zeit aufgebraucht werden.

Frühzeitig müssen deswegen weitere wichtige Faktoren für die Aufrechterhaltung der Versorgung adressiert werden:

- Aufstockung der Bevorratung

- Sicherstellung von Lieferketten

- Sicherung des Bestands

Bereits vor dem Auftreten erwartbarer Infektionsfälle wurde die Bevorratung insbesondere von Atemschutzmasken aufgestockt. Der zusätzliche Puffer dient dazu, kurzfristig auftretende Bedarfsspitzen bzw. Lieferverzögerungen abfangen zu können.

Vor dem Hintergrund möglicher Versorgungsengpässe ist es umso wichtiger, bestehende Ressourcen so gezielt wie möglich einzusetzen und frühzeitigen Verlusten durch unverhältnismäßigen Gebrauch bzw. Bevorratung in den Bereichen sowie Diebstahl vorzubeugen. Dies wurde erreicht, indem Schutzkleidung und Desinfektionsmittel zentral gesichert und eine Belieferung dezentraler Verbraucher auf die unmittelbar in die Versorgung möglicher infizierter Patienten eingebundenen Bereiche beschränkt wurde. Die Ausgabe erfolgt nur auf Begründung des Bedarfs und entsprechende Gegenzeichnung hin. Der ressourcensparende Einsatz der persönlichen Schutzausrüstung auch bei anderen Infektionserkrankungen wurde in alle Bereiche kommuniziert.

\section{Fazit für die Praxis}

Schon in der Initialphase einer möglichen Pandemie ist es für das Gesamtklinikum und im Speziellen für die Notaufnahme als Dreh- und Angelpunkt von Patientenidentifikation, Screening und Erstdiagnostik von entscheidender Bedeutung, dass Experten aus den Bereichen der Patientenversorgung, Diagnostik, Hygiene und Kommunikation im engen Austausch die aktuelle Lage abschätzen und schnellstmögliche Lösungen und Prozessänderungen erarbeiten. Hierzu zählen die Organisation einer frühen Trennung der Patienten, Implementierung von Screeningalgorithmen, Schaffung von Isolierstationen/Betten und Aufstockung der Intensivstationskapazitäten.

Neben den klinischen Gesichtspunkten sind eine strukturierte Steuerung, Kommunikation und Exekution auf allen Ebenen des Klinikums eine Grundvoraussetzung, um schnell handlungsfähig zu bleiben.

\section{Korrespondenzadresse}

\section{Dr. med. Michael Dommasch}

Fakultät für Medizin, Zentrale Interdisziplinäre Notaufnahme, Klinikum rechts der Isar, Technische Universität München Ismaninger Str. 22, 81675 München, Deutschland

michael.dommasch@mri.tum.de

\section{Einhaltung ethischer Richtlinien}

Interessenkonflikt. M. Dommasch, F. Gebhardt, U. Protzer, A. Werner, E. Schuster, C. Brakemeier, J. Mayer, S. Feihl, C. Querbach, R. Braren, M. Treiber, F. Geisler und C.D. Spinner geben an, dass kein Interessenkonflikt besteht.

Für diesen Beitrag wurden von den Autoren keine Studien an Menschen oder Tieren durchgeführt. Für die aufgeführten Studien gelten die jeweils dort angegebenen ethischen Richtlinien.

\section{Literatur}

1. WHO (2020) Statement on the second meeting of the International Health Regulations (2005) Emergency Committee regarding the outbreak of novel coronavirus (2019-nCoV). https://www.who. int/news-room/detail/30-01-2020-statementon-the-second-meeting-of-the-international- health-regulations-(2005)-emergency-committee-regarding-the-outbreak-of-novel-coronavirus-(2019-ncov). Zugegriffen:29. Juni 2020

2. Wu Z, McGoogan JM (2020) Characteristics of and important lessons from the coronavirus disease 2019 (COVID-19) outbreak in China. JAMA. https:// doi.org/10.1001/jama.2020.2648

3. Rothe C, Schunk M, Sothmann P, Bretzel G, Froeschl G, Wallrauch C, Zimmer T, Thiel V, Janke C, Guggemos W, Seilmaier M, Drosten C, Vollmar P, Zwirglmaier K, Zange S, Wölfel R, Hoelscher M (2020) Transmission of 2019-nCoV infection from an asymptomatic contact in Germany. N Engl J Med 382(10):970-971

4. https://www.rki.de/DE/Content/InfAZ/N/ Neuartiges_Coronavirus/Risikobewertung. html.Zugegriffen: 29. Juni 2020

5. Gu J, Han B, Wang J (2020) COVID-19: Gastrointestinal manifestations and potential fecal-oral transmission. Gastroenterology. https://doi.org/ 10.1053/j.gastro.2020.02.054

6. Chang D, Lin M, Wei L, Xie L, Zhu G, Dela Cruz CS, Sharma L (2020) Epidemiologic and clinical characteristics of novel coronavirus infections involving 13 patients outside Wuhan, China.JAMA. https://doi.org/10.1001/jama.2020.1623

7. Xie C, Jiang L, Huang G, Pu H, Gong B, Lin H, Ma S, Chen X, Long B, Si G, Yu H, Jiang L, Yang X, Shi Y, Yang Z (2020) Comparison of different samples for 2019 novel coronavirus detection by nucleic acid amplification tests. Int J Infect Dis. https://doi.org/ 10.1016/j.jijid.2020.02.050

8. Shi H, Han X, Jiang N, Cao Y, Alwalid O, Gu J, Fan Y, Zheng C (2020) Radiological findings from 81 patients with COVID-19 pneumonia in Wuhan, China: a descriptive study. Lancet Infect Dis. https://doi.org/10.1016/S1473-3099(20)30086-4

9. Zhang J, Zhou L, Yang Y, Peng W, Wang W, Chen X (2020) Therapeutic and triage strategies for 2019 novel coronavirus disease in fever clinics. Lancet RespirMed 8(3):e11-e12

10. Zhang W, Du RH, Li B, Zheng XS, Yang XL, Hu B, Wang YY, Xiao GF, Yan B, Shi ZL, Zhou P (2020) Molecular and serological investigation of 2019-nCoV infected patients: implication of multiple shedding routes. Emerg Microbes Infect 9(1):386-389

11. Wölfel R, Corman VM, Guggemos W, Seilmaier M, Zange S, Müller MA, Niemeyer D, Jones TC, Vollmar P, Rothe C, Hoelscher M, Bleicker T, BrüninkS, Schneider J, Ehmann R, Zwirglmaier K, Drosten C, Wendtner C (2020) Virological assessment of hospitalized patients with COVID-2019. Nature 581:465-469. https://doi.org/10.1038/s41586020-2196-x

12. Cobos AJ, Nelson CG, Jehn M, Viboud C, Chowell G (2016) Mortality and transmissibility patterns of the 1957 influenza pandemic in Maricopa County, Arizona. BMC Infect Dis 16(1):405

13. GräffI, Goldschmidt B, Glien P, Bogdanow M (2014) The German Version of the Manchester Triage System and its quality criteria-first assessment of validity and reliability. Plos One. https://doi.org/ 10.1371/journal.pone.0088995

14. https://www.rki.de/DE/Content/InfAZ/N/ Neuartiges_Coronavirus/Ergaenzung_Pandemie plan_Covid.pdf?_blob=publicationFile. Zugegriffen:29. Juni 2020

15. https://www.rki.de/DE/Content/Infekt/Prepared ness_Response/Rahmenkonzept_Epidemische bedeutsame_Lagen.pdf?_blob=publicationFile. Zugegriffen: 29. Juni 2020 
16. Lenzen-Schulte M (2020) Bei COVID-19-Verdacht: Rasche Triage symptomatischer Patienten in der Notaufnahme. Dtsch Arztebl 117(14):A-715

17. https://www.baua.de/DE/Angebote/Aktuelles/ Meldungen/2020/2020-02-19-Coronavirus.html. Zugegriffen:29. Juni 2020

\section{Strategien zur Verbesserung der Kommunikation bei Schwerhörigkeit, insbesondere beim Tragen eines Mund- Nasen-Schutzes}

„Verstehen mich mit der Maske eigentlich noch alle?" Unter diesem Titel behandeln Prof. Maria Schuster (Nürnberg und München) und Koautoren aus der Sicht der Phoniatrie/Audiologie in Zusammenwirken mit Allgemeinmedizin und Informatik Probleme der verbalen Kommunikation beim Tragen eines Mund-Nasen-Schutzes.

Bei mehr als $30 \%$ der über 70 -Jährigen muss man mit Hörproblemen rechnen, und auch bei versorgter Schwerhörigkeit kann noch eine Beeinträchtigung des Sprachverstehens vorliegen. Zusätzlich vermindert der MundNasen-Schutz, obligatorisch in Zeiten von Corona, die Sprachqualität, durch ein lauteres Sprechen kann man dieses nicht ausgleichen, da es durch die Maske zu einer akustischen Veränderung der Sprache kommt. Auch verdeckt der Mund-Nasen-Schutz weite Teile des Gesichtes, so dass die Ausdruckskraft der Mimik weitgehend entfällt und Worte nicht mehr von den Lippen abgelesen werden können; eine durchsichtige Maske kann dieses Problem mindern.

Deshalb gilt es gerade jetzt, die eigenen Strategien für das Gespräch mit älteren Patienten, bei denen man mit einer Schwerhörigkeit rechnen kann, zu überprüfen und gegebenenfalls durch geschriebene Informationen zu erweitern.

So formulieren die Autoren als Fazit für die Praxis:

1. Eine Mund-Nasen-Maske kann die Sprachqualität deutlich vermindern.

2. Bei hochfiltrierenden Masken ist dieser Effekt noch größer als bei chirurgischen Masken.

3. Insbesondere bei Patienten mit Hörschädigung sollte man sich dessen bewusst sein und gute Voraussetzungen für die Kommunikation schaffen.

4. Helfen können vor allem eine gut ausgeleuchtete, geräuscharme Umgebung, die Gestik des Sprechenden, eine schriftliche Zusammenfassung sowie gegebenenfalls ein Gebärdendolmetscher.

5. Lauteres Sprechen oder drahtlose Übertragungsanlagen verbessern nicht die Qualität der Sprache, für das Sprechen ohne Maske fehlt meistens der Platz zum Abstand halten.
Anmerkung des Rezensenten: Darüber hinaus könnte es sinnvoll sein, bei (Erst-)Aufnahme der Anamnese zu fragen: Tragen Sie ein Hörgerät?

Quelle (Der Beitrag ist frei zugänglich): Schuster M, Arias-Vergara T, Müller-Hörner R, Winterholler C, Bocklet T (2020) Verstehen mich mit der Maske eigentlich noch alle? Geriatrie-Report 15 (3): 8-9.

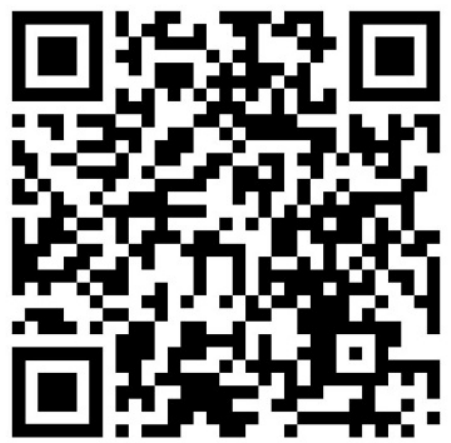

Einfach einscannen und direkt Beitrag lesen

Rezensent: Dr. Udo Hennighausen, Augenärztliche Gemeinschaftspraxis, Markt 9, 25746 Heide, E-Mail: Udo.Hennighausen@web.de 